\title{
Resonant dynamics in boson-fermion mixtures
}

\author{
M. Wouters, J. Tempere, ${ }^{*}$ and J. T. Devreese ${ }^{\dagger}$ \\ TFVS, Universiteit Antwerpen, Universiteitsplein 1, B2610 Antwerpen, Belgium \\ (Received 31 January 2003; published 25 June 2003)
}

\begin{abstract}
The time evolution of a dilute atomic boson-fermion mixture is studied at zero temperature, when the external magnetic field is brought close to an interspecies Feshbach resonance. On short time scales we find that before a collapse or phase separation of the mixture can occur, oscillations between fermionic atoms and heteronuclear molecules dominate the dynamics. Applying the model presented here to a trapped mixture of ${ }^{40} \mathrm{~K}-{ }^{87} \mathrm{Rb}$ atoms, we find that the predicted oscillations can be revealed in degenerate mixtures of these atoms.
\end{abstract}

DOI: 10.1103/PhysRevA.67.063609

PACS number(s): 03.75.Kk, 05.30.Fk, 05.30.Jp, 32.80.Pj

\section{INTRODUCTION}

One of the most attractive features of cold atomic gases is that the interatomic scattering length is under very good experimental control. Using the presence of Feshbach resonances, the scattering length can be tuned from a highly repulsive to a strongly attractive one by changing the applied magnetic field. Sympathetic cooling techniques make it possible to cool mixtures of fermionic and bosonic atoms down to extremely low temperatures where the quantummechanical nature of both bosons and fermions becomes apparent, resulting in a Bose-Einstein condensate coexisting with a filled Fermi sea $[1,2]$. When the interspecies interactions are strongly attractive, changing the number of bosons alters the mean-field interaction strength and can result in a collapse of the Fermi gas as observed by Modugno et al. [3]. This instability can also be triggered by changing the scattering length itself, making use of a Feshbach resonance. The divergence of the scattering length at a Feshbach resonance is due to a magnetically tunable molecular state whose binding energy vanishes at resonance. This means that close to this magnetic-field value, the molecular state can be populated. A number of experimental [4] and theoretical works [5-8] have been devoted to study the conversion of atoms between the atomic and molecular condensates and the thermal cloud.

In this work we study the evolution of an initially noninteracting boson-fermion mixture at zero temperature when a sudden change (on a time scale much faster than any other time scale in the system) in the magnetic field brings it close to an interspecies Feshbach resonance. The (fermionic) molecules first form out of a condensate boson and a fermionic atom with an energy smaller than or equal to the Fermi energy. We will refer to such fermionic atoms (molecules) as "slow" fermions (molecules); and to fermions or molecules with more energy than the Fermi energy as "fast" fermions or molecules, respectively. The decay of a molecule can then either lead back to a condensate boson with the same slow

\footnotetext{
*Also at Lyman Laboratory of Physics, Harvard University, Cambridge, Massachusetts 02138.

${ }^{\dagger}$ Also at Universiteit Antwerpen (RUCA), Groenenborgerlaan 171, B-2020 Antwerpen, Belgium and Technische Universiteit Eindhoven, P. B. 513, 5600 MB Eindhoven, The Netherlands.
}

fermion or to a thermal boson accompanied by a fast fermion. Energy conservation is not violated because the interaction energy is changed. The latter process shows that the stepwise change of the magnetic field can lead to heating of the system, while the former demonstrates that coherent oscillations between fermionic atoms and molecules are possible when a fermionic atom interacts with the condensate as a whole. Due to multiple scattering of fermionic atoms, bosons, and molecules, the time evolution of the fast part of the system is expected to be rather chaotic. This is why we preferred to focus on the slow fermionic atoms and molecules. The energies involved in the formation of molecules are easily two orders of magnitude greater than the Fermi energy, and thus we conclude from the difference in phasespace volume that it is rather unlikely that the fast decay products of a molecule will come back in the Fermi sphere. This assumption allows us to study the slow fermionic atoms and molecules as a subsystem.

Our study is based on the model Hamiltonian introduced in Sec. II. We make some approximations that allow us to write the time dependence of slow fermionic atoms and molecules in an analytical form. The results obtained with this simplified model are discussed in Sec. III, together with the experimental conditions needed to make the predictions observable, using the data from ${ }^{40} \mathrm{~K}-{ }^{87} \mathrm{Rb}$ scattering.

\section{DESCRIPTION OF THE DYNAMICS}

Experimentally, the scattering length as a function of the magnetic field close to a Feshbach resonance can be well parametrized by

$$
a_{\text {scatt }}(B)=a_{b g}\left(1-\frac{\Delta B}{B-B_{0}}\right),
$$

where $a_{b g}$ is the background scattering length, $B_{0}$ is the position, and $\Delta B$ is the width of the resonance.

A good description of the scattering physics near a Feshbach resonance can be obtained by introducing molecules explicitly $[9,10]$. These molecular states are the bound states of an interatomic potential in a closed channel, and their energy relative to the threshold is given by

$$
\nu(B)=\left(B-B_{0}\right) \Delta \mu,
$$

where $\Delta \mu=\mu^{\text {molecule }}-\mu^{\text {fermion }}-\mu^{\text {boson }}$ is the difference in magnetic moment between the molecular and atomic states. 
Denoting the coupling strength between atoms and molecules by $g_{m}$, the Hamiltonian for a trapped gas can be written as

$$
\begin{aligned}
H= & \int d^{3} \mathbf{x}\left[\psi_{f}^{\dagger}(\mathbf{x}) H_{f}^{0}(\mathbf{x}) \psi_{f}(\mathbf{x})+\psi_{b}^{\dagger}(\mathbf{x}) H_{b}^{0}(\mathbf{x}) \psi_{b}(\mathbf{x})\right. \\
& +\psi_{m}^{\dagger}(\mathbf{x}) H_{m}^{0}(\mathbf{x}) \psi_{m}(\mathbf{x})+g_{m} \psi_{m}^{\dagger}(\mathbf{x}) \psi_{f}(\mathbf{x}) \psi_{b}(\mathbf{x}) \\
& \left.+g_{m}^{*} \psi_{b}^{\dagger}(\mathbf{x}) \psi_{f}^{\dagger}(\mathbf{x}) \psi_{m}(\mathbf{x})\right],
\end{aligned}
$$

where $\quad H_{f, b}^{0}(\mathbf{x})=-\hbar^{2} \nabla^{2} / 2 m_{f, b}+V_{\text {trap }}^{f, b}(\mathbf{x}) \quad$ and $\quad H_{m}^{0}(\mathbf{x})$ $=-\hbar^{2} \nabla^{2} / 2 m_{m}+V_{\text {trap }}^{m}(\mathbf{x})+\nu_{\text {bare }}(B)$ are the Hamiltonians of the noninteracting gases of fermionic atoms, bosons, and molecules, respectively. The corresponding masses of these atoms and molecules, and the trapping potentials confining them are indexed by $f, b$, and $m$ to indicate fermionic atoms, bosons, and molecules, respectively. In the last term we stress that the parameter $\nu_{\text {bare }}$ appearing in Hamiltonian (3) is the bare molecular binding energy [10]. The true binding energy $\nu(B)$ given in Eq. (2) differs from this quantity due to virtual dissociations of the molecules. Background scattering is omitted, because we assume that the system is brought close enough to resonance, so that the resonant contribution is much more important.

Starting from Hamiltonian (3), we wish to study the dynamics of the mixture when the scattering length is suddenly brought close to a Feshbach resonance. In order to grasp the basic physics, some approximations are made. First of all we assume that there are much more condensate atoms than fermions, so that the effect on the condensate can be neglected. Also the depletion of the condensate is presumed to be small, which allows for the Bogoliubov approximation. Finally, we assume that the distance that a particle travels during a molecule formation process is small compared to the harmonicoscillator length. The latter permits us to restrict ourselves to homogeneous systems at first and use a local-density approximation to take the inhomogeneity due to the confinement potential into account at a later stage. All these assumptions will be justified in Sec. III.

We thus consider a homogeneous boson-fermion mixture without interactions before $t=0$, the moment when the magnetic field is suddenly brought close to resonance and the interactions between the atoms start taking place. At $t \leqslant 0$, the initial state consists of condensate bosons, slow fermionic atoms (filling the Fermi sphere), and no molecules. From $t=0$ onwards, the evolution of this mixture is described by Eq. (3). The evolution will consist in the formation of molecules and their subsequent decay. Pauli blocking will limit the decay channels. However, the molecular binding energy $\nu$ is, even for magnetic fields that are only $0.1 \mathrm{G}$ away from resonance, much larger than the Fermi energy [since $\nu=(0.1 \mathrm{G})\left(\mu_{B} / k_{B}\right) \approx 6.7 \mu \mathrm{K}$, with $\mu_{B}$ and $k_{B}$ the Bohr magneton and the Boltzmann constant, respectively, whereas $E_{F}$ is of the order of a few hundred $\mathrm{nK}$ ], and blocking effects will be small. As a consequence, a single-particle description of the fermions should be a good first approximation. The simplified Hamiltonian for this fermion-boson mixture reads

$$
\begin{aligned}
H= & \int \frac{d^{3} \mathbf{k}}{(2 \pi)^{3}}\left[\frac{\hbar^{2} k^{2}}{2 m_{f}} \psi_{f}^{\dagger}(\mathbf{k}) \psi_{f}(\mathbf{k})+\frac{\hbar^{2} k^{2}}{2 m_{b}} \psi_{b}^{\dagger}(\mathbf{k}) \psi_{b}(\mathbf{k})\right. \\
& \left.+\left(\frac{\hbar^{2} k^{2}}{2 m_{m}}+\nu_{\text {bare }}\right) \psi_{m}^{\dagger}(\mathbf{k}) \psi_{m}(\mathbf{k})\right] \\
& +g_{m} \sqrt{n_{b}} \int \frac{d^{3} \mathbf{k}}{(2 \pi)^{3}} \psi_{m}^{\dagger}(\mathbf{k}) \psi_{f}(\mathbf{k}) \\
& +g_{m} \int \frac{d^{3} \mathbf{k}_{f}}{(2 \pi)^{3}} \frac{d^{3} \mathbf{k}_{b}}{(2 \pi)^{3}} \psi_{m}^{\dagger}\left(\mathbf{k}_{f}+\mathbf{k}_{b}\right) \psi_{f}\left(\mathbf{k}_{f}\right) \psi_{b}\left(\mathbf{k}_{b}\right)+\text { H.c. }
\end{aligned}
$$

Here, $g_{m} \sqrt{n_{b}}$ has the dimension of energy and we will use

$$
\tau=\operatorname{tg}_{m} \sqrt{n_{b}} / \hbar \quad \text { and } \quad \tilde{m}=g_{m} m_{r} /\left(\hbar^{2} n_{b}^{1 / 6}\right)
$$

as our dimensionless quantities, with $m_{r}=\left(m_{f}^{-1}+m_{b}^{-1}\right)^{-1}$ the reduced mass of a fermion-boson pair. The first line in Eq. (4) represents the free gases of fermionic atoms, bosons, and molecules, respectively. The second line in Eq. (4) describes the interaction converting a fermionic atom and a boson into a molecule (slow or fast) and vice versa. In the single-particle picture, we should study the time evolution of each fermion in the Fermi sea individually. However, because the Fermi level is much smaller than the molecular binding energy, the initial kinetic energy of the slow fermion does not influence the final result for the dynamics and can be neglected. Consequently, all the slow fermions will have a similar time evolution due to the interaction with the condensate, irrespective of the specific value of their momentum. Thus, it will suffice to analyze the properties of a fermion with initial momentum $k=0$ to know the evolution of all of them.

An appropriate wave function to describe one fermion that is initially at rest is

$$
\begin{aligned}
|\psi(t)\rangle= & {\left[f(t) \psi_{f}^{\dagger}\left(k_{f}=0\right)|0\rangle+m(t) \psi_{m}^{\dagger}\left(k_{m}=0\right)|0\rangle\right.} \\
& \left.+\int d^{3} \mathbf{k}_{f} w\left(k_{f}, t\right) \psi_{f}^{\dagger}\left(\mathbf{k}_{f}\right) \psi_{b}^{\dagger}\left(-\mathbf{k}_{f}\right)|0\rangle\right]\left|N_{b}\right\rangle,
\end{aligned}
$$

where $f(t)$ and $m(t)$ represent the amplitude of the bare fermionic atom and the bare molecule. The third term, containing the amplitude $w\left(k_{f}, t\right)$, arises from molecules that dissociate in a fast fermionic atom and a thermal boson, which is an important process. Higher order processes, where the fast fermionic atom again forms a molecule that dissociates, are neglected in this approach. Diagrammatically speaking, this comes down to retaining only the diagrams of Fig. 1(a), and neglecting the contributions like those in Fig. 1(b).

The Schrödinger equation $i \hbar \partial_{t}|\psi\rangle=H|\psi\rangle$ allows us to find the time evolution of the amplitudes in wave function (6):

$$
i \hbar \frac{d}{d t} f(t)=g_{m} \sqrt{n_{b}} m(t)
$$




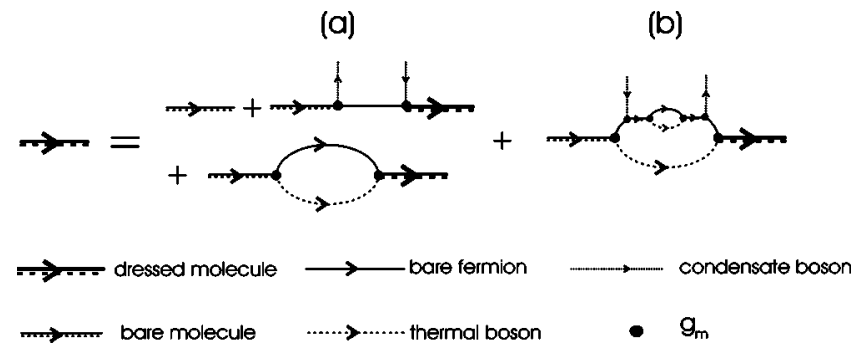

FIG. 1. Feynman diagrams that describe the correction to the Green function of the heteronuclear molecules in the mixture. The terms in the left part (a) are taken into account in the theory of this paper. Multiple dissociations as depicted in the right part (b) are neglected.

$$
\begin{gathered}
i \hbar \frac{d}{d t} m(t)=\nu_{\text {bare }}+g_{m} \sqrt{n_{b}} f(t)+g_{m} \int \frac{d^{3} \mathbf{k}_{f}}{(2 \pi)^{3}} w\left(k_{f}, t\right) \\
i \hbar \frac{d}{d t} w\left(k_{f}, t\right)=\frac{\hbar^{2} k_{f}^{2}}{m_{r}} w\left(k_{f}, t\right)+g_{m} m(t)
\end{gathered}
$$

We choose as initial conditions $f(t=0)=1$ and $m(t=0)$ $=w\left(k_{f}, t=0\right)=0$. This corresponds to the initial state wave function describing one slow fermionic atom, $\left|\psi_{\text {init }}\right\rangle$ $=\psi_{f}^{\dagger}\left(k_{f}=0\right)|0\rangle$. Note that $\sqrt{n_{b}}$ is assumed time independent. The linear-differential equations (7)-(9) can be solved with a Laplace transform, yielding

$$
\begin{aligned}
f(\tau)= & \frac{1}{\omega_{0}\left(2 \omega_{0}+\tilde{\nu}+\frac{3}{8 \pi}(2 \tilde{m})^{3 / 2} \sqrt{\omega_{0}}\right)} e^{i \omega_{0} \tau} \\
& +\int_{0}^{\infty} e^{-i \omega \tau} \frac{1}{4 \pi^{2}} \frac{(2 \tilde{m})^{3 / 2} \sqrt{\omega}}{[\omega(\omega-\tilde{\nu})-1]^{2}+\frac{(2 \tilde{m} \omega)^{3}}{16 \pi^{2}}} d \omega,
\end{aligned}
$$

and

$$
\begin{aligned}
m(\tau)= & \frac{-1}{2 \omega_{0}+\tilde{\nu}+\frac{3}{8 \pi}(2 \tilde{m})^{3 / 2} \sqrt{\omega_{0}}} e^{i \omega_{0} \tau} \\
& +\int_{0}^{\infty} e^{-i \omega \tau} \frac{1}{4 \pi^{2}} \frac{(2 \tilde{m} \omega)^{3 / 2}}{[\omega(\omega-\tilde{\nu})-1]^{2}+\frac{(2 \tilde{m} \omega)^{3}}{16 \pi^{2}}} d \omega,
\end{aligned}
$$

where $\omega_{0}$ is a root of $\omega\left[\omega+\tilde{\nu}+(1 / 4 \pi)(2 \tilde{m})^{3 / 2} \sqrt{\omega}\right]-1=0$ and $\tilde{\nu}$ is a dimensionless parameter related to the real molecular binding energy $\nu$ by $\tilde{\nu}=\nu /\left(g_{m} \sqrt{n_{b}}\right)$. In evaluating the integral in Eq. (8), we have used the relation between the real and bare molecular binding energy, $\nu$ and $\nu_{\text {bare }}$, for a momentum cutoff $K[10]: \nu=\nu_{\text {bare }}-K g_{m}^{2} m_{r} /(\pi \hbar)^{2}$.

\section{RESULTS AND DISCUSSION}

\section{A. Atom-molecule oscillations}

It is instructive for the understanding of the time evolution to investigate the eigenstates of Hamiltonian (4) describing the system in which the time evolution takes place. The ground state for a single fermionic atom in a system described by Eq. (4) can be investigated by using Eq. (6) as a variational wave function with $f, m, w\left(k_{f}\right)$ as variational parameters. The variational expression for the ground-state energy is

$$
\begin{aligned}
\frac{E_{0}\left(f, m, w\left(k_{f}\right)\right)}{g_{m} \sqrt{n_{b}}}= & \left(f^{*} m+m^{*} f\right)+\int \frac{d^{3} \mathbf{k}_{f}}{(2 \pi)^{3}} \frac{k_{f}^{2}}{2 \tilde{m}}\left|w\left(k_{f}\right)\right|^{2} \\
& +\int \frac{d^{3} \mathbf{k}_{f}}{(2 \pi)^{3}}\left[m^{*} w\left(k_{f}\right)+w^{*}\left(k_{f}\right) m\right]
\end{aligned}
$$

where $k_{f}$ is now measured in units $n_{b}^{1 / 3}$. Minimizing this expression, with the constraint $|\langle\psi \mid \psi\rangle|=1$, we find for the ground-state energy the equation

$$
\widetilde{E}\left(\tilde{E}-\tilde{\nu}-\int \frac{d^{3} \mathbf{k}_{f}}{(2 \pi)^{3}} \frac{1}{\widetilde{E}-\frac{k_{f}^{2}}{2 \tilde{m}}}\right)-1=0 .
$$

Here $\widetilde{E}=E /\left(g_{m} \sqrt{n_{b}}\right)$, which has as lowest solution (relative to the bottom of the fermion band) $\widetilde{E}=-\omega_{0}$. The ground state is a bound state in the sense that it has negative energy with respect to the energy of a free fermionic atom. It is interesting to note that for $\tilde{\nu}>0$, the ground-state energy is a descreasing function of $n_{b}$. This might signal the collapse of the boson-fermion mixture for attractive interactions. The bound state represents a coherent superposition of the states corresponding to the fermion as an atom and as a part in a molecule:

$$
\begin{aligned}
\left|\psi_{\text {bound }}\right\rangle= & \mathcal{N}\left[\frac{-1}{\omega_{0}} \psi_{f}^{\dagger}\left(k_{f}=0\right)+\psi_{m}^{\dagger}\left(k_{m}=0\right)\right. \\
& \left.+\int \frac{d^{3} \mathbf{k}_{f}}{(2 \pi)^{3}} \frac{1}{-\omega_{0}-\frac{k_{f}^{2}}{2 \tilde{m}}} \psi_{f}^{\dagger}\left(\mathbf{k}_{f}\right) \psi_{b}^{\dagger}\left(-\mathbf{k}_{f}\right)\right]|0\rangle,
\end{aligned}
$$

with $\mathcal{N}$ a normalization constant. Similar bound states are found in the photodissociation of a negative ion [11]. Equation (13) has only one solution for $E$ negative. The solutions of Eq. (13) with $\widetilde{E}=\omega>0$ form a continuum, because we 


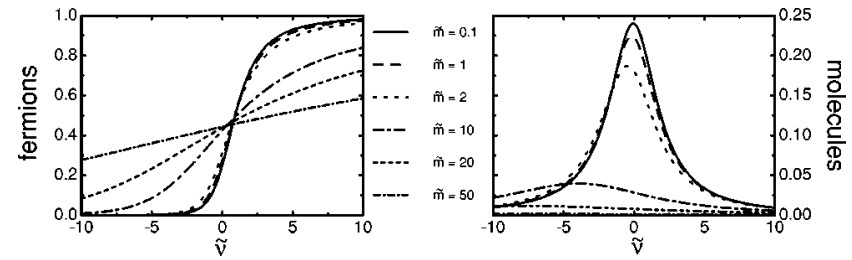

FIG. 2. The fraction of slow molecules $|m(t)|^{2}$ [expression (11)] and the fraction slow fermionic atoms $|f(t)|^{2}$ [expression (10)] are shown in the right and left panels, respectively, for $t \rightarrow \infty$ as a function of the molecular binding energy $\tilde{\nu}$ for different values of the mass $\tilde{m}$.

consider the limit of an infinite system. The eigenstates $\left|\psi_{\text {exc }}(\omega)\right\rangle$ corresponding to these solutions are the excited states of Hamiltonian (4).

The time evolution of the initial state $\left|\psi_{\text {init }}\right\rangle$ can then be written in the basis of the eigenstates of Eq. (4) as

$$
\begin{aligned}
|\psi(t)\rangle=\left|\psi_{\text {bound }}\right\rangle e^{i \omega_{0} t}\left\langle\psi_{\text {bound }} \mid \psi_{\text {init }}\right\rangle \\
\quad+\int_{0}^{\infty}\left|\psi_{\text {exc }}(\omega)\right\rangle e^{-i \omega t}\left\langle\psi_{\text {exc }}(\omega) \mid \psi_{\text {init }}\right\rangle d \omega .
\end{aligned}
$$

In Fig. 2, the modulus square of $\left\langle\psi_{\text {bound }} \mid \psi_{\text {init }}\right\rangle$, is shown for several values of $\tilde{\nu}$ in the left panel. In the right panel, the modulus square of the projection of the bound state on the molecular state, $\left|\left\langle\psi_{\text {bound }}\left|\psi_{m}^{\dagger}\left(k_{m}=0\right)\right| 0\right\rangle\right|^{2}$, is shown. Note that expression (15) has the same structure as expressions (10) and (11). This is to be expected because the amplitudes $f(t)$ and $m(t)$ represent the projection of $|\psi(t)\rangle$ on the slow fermionic atom component and the slow molecule component:

$$
\begin{gathered}
f(t)=\left\langle 0\left|\psi_{f}\left(k_{f}=0\right)\right| \psi(t)\right\rangle, \\
m(t)=\left\langle 0\left|\psi_{m}\left(k_{f}=0\right)\right| \psi(t)\right\rangle .
\end{gathered}
$$

The two terms appearing in $|\psi(t)\rangle$, expression (15), give rise to the corresponding two terms in $f(t)$ [expression (10)] and in $m(t)$ [expression (11)]. The first term [the first line in expressions(10) and (11)] is related to the bound state and the second term (containing the integration over $\omega$ ) is related to the excited states. Because of the continuous character of the excited states as a function of $\omega$, the terms related to the excited states vanish for large times.

We find that for large $|\widetilde{\nu}|$, the conversion from slow to fast fermions is greatly reduced. For $\tilde{\nu}<0$, this can be understood from the fact that $\left\langle\psi_{\text {bound }} \mid \psi_{\text {init }}\right\rangle$ becomes very small (as can be seen in Fig. 2) and the initial state coincides nearly with an eigenstate of the system, $\left|\psi_{\text {init }}\right\rangle \approx \mid \psi_{\text {exc }}(\omega$ $=0)\rangle$, so that $|\psi(t)\rangle \approx\left|\psi_{\text {init }}\right\rangle$ and $f(t)$ remains close to 1 (its initial value).

The fraction of slow molecules or slow fermionic atoms, obtained from Eqs. (10) and (11), respectively, displays damped oscillations resulting from the interference between the terms related to the bound state and the terms related to
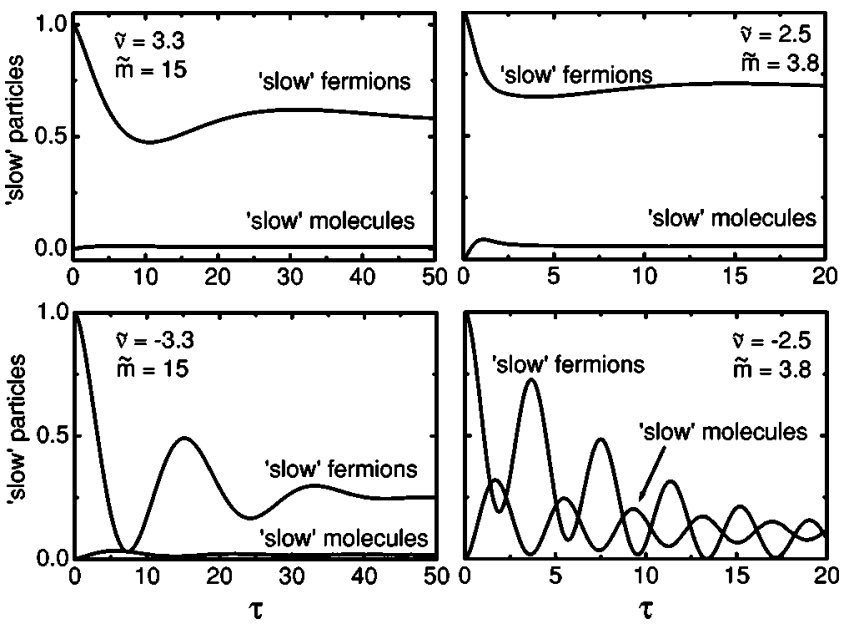

FIG. 3. The time evolution of the fractions of slow fermionic atoms, $|f(t)|^{2}$ and molecules, $|m(t)|^{2}$, given by expressions (10) and (11), are shown for two different values of the molecular binding energy and the mass. The molecular binding energies correspond to detunings of the magnetic field of $B-B_{0}= \pm 0.1 \mathrm{G}$. For $n_{b}=10^{14} \mathrm{~cm}^{-3}$, the dimensionless detunings and the masses correspond to the predicted ${ }^{40} \mathrm{~K}-{ }^{87} \mathrm{Rb}$ resonances in the $(9 / 2,-9 / 2)$ $+(1,1)$ hyperfine states at $741.2 \mathrm{G}$ (left) and to the $(9 / 2,-7 / 2)$ $+(1,1)$ at $522.9 \mathrm{G}$ (right).

the excited states. Figure 3 shows the evolution of the densities of slow fermionic atoms and slow molecules for different values of the molecular binding energy $\tilde{\nu}$ and mass $\tilde{m}$. For the chosen values of the mass, the oscillations are visible for negative molecular binding energy $\tilde{\nu}<0$, while for positive $\tilde{\nu}$ they are strongly damped.

We observed that the larger the $\tilde{m}$, the fewer molecules are formed. Indeed, according to Fermi's golden rule, a bigger reduced mass means a faster dissociation rate $\left(\sim m_{r}^{3 / 2}\right)$ of the molecules. Remarkably, also for $\tilde{m} \gg 1$, the fraction of slow fermions reduces substantially over time even though at all times few molecules that can dissociate to fast fermionic atoms are present. This effect can be understood, since even though few molecules are present at any time, they form and dissociate quickly. This raises the question whether our approximate wave function (6) is good enough. For low $\tilde{m}$, it is obvious that the dissociation of molecules will be slow, and strong Rabi oscillations between fermionic atoms and molecules occur. The third term of Eq. (6) [containing the factor $\left.w\left(k_{f}, t\right)\right]$, is then relatively unimportant, and corrections on it will not affect the result a lot. For high $\tilde{m}$, the molecules dissociate rapidly and the contribution to $|\psi(t)\rangle$ coming from the pairs of fast fermionic atoms and bosons [last term in Eq. (6)] must be big. But in this case the corrections to the contribution of these pairs - through new formation of fast molecules out of a fast fermionic atom-are small, because few of those molecules are formed. Diagrammatically, this means that diagrams, such as those in Fig. 1(b), are negligible. Then for intermediate $\tilde{m}$ one can also expect Eq. (6) to be a good approximation, at least in order to calculate the fraction of slow fermions. 


\section{B. Relation to experiments on ${ }^{40} \mathrm{~K}-{ }^{87} \mathrm{Rb}$ mixtures}

To check whether the picture described here, with fermions that form a bound state with the condensate, is correct, one has to be able to measure preferably the fraction of slow fermionic atoms. This should, after some transient behavior, go to a constant value and not to zero. They can be detected by a time-of-flight expansion after a magnetic pulse has been applied.

In ${ }^{40} \mathrm{~K}-{ }^{87} \mathrm{Rb}$, plenty of Feshbach resonances have been predicted $[12,13]$, allowing us to estimate quantitatively the magnitude of the model parameters. Therefore, we make use of $g_{m}=\sqrt{2 \pi \hbar^{2} a_{b g} \Delta B \Delta \mu / m_{r}}$, with $a_{b g}$ the background scattering length. As an example, we take the resonance between the $(9 / 2,-9 / 2)+(1,1)$ hyperfine states around $741.2 \mathrm{G}$. For $n_{b}=10^{14} \mathrm{~cm}^{-3}$, the frequency $g_{m} \sqrt{n_{b}} / \hbar$ is equal to 7.72 $\times 10^{5} \mathrm{~Hz}$, which is much larger than the harmonic-oscillator frequency. Thus we can expect that the confinement is relatively unimportant for the atom-molecule oscillations. We find in this case $\tilde{\nu}=33.13\left[\left(B-B_{0}\right)(\mathrm{G})\right] / \sqrt{n_{b}\left(10^{14} \mathrm{~cm}^{-3}\right)}$, and further, $\tilde{m}=15.47$. The values used for the parameters of Fig. 3 are for this resonance with $n_{b}=10^{14} \mathrm{~cm}^{-3}$ and $B-B_{0}= \pm 0.1 \mathrm{G}$ on the left, and on the right for the $(9 / 2,-7 / 2)+(1,1)$ hyperfine states with a Feshbach resonance around $522.9 \mathrm{G}$ [14].

The current results have been derived in the framework of a translation invariant system, whereas in the experimental setup the inhomogeneity of the trapped gases is apparent. The time evolution has been calculated for homogeneous mixtures, and it is necessary to estimate what the effects due to the confinement are. Considering the case with much more bosons than fermions, we can neglect in a first approximation the change of the boson density. Then one can find in a mean-field approximation the redistribution speed of the fermions in the trap. The local-density approximation is applicable only if during the time scale of a Rabi oscillation between the fermionic atom state and the molecular state, the fermions have not moved over a substantial fraction of the trap length. In the Thomas-Fermi approximation for the bosons, the mean-field potential felt by a fermion is $\left(2 \pi \hbar^{2} a_{\text {scatt }} n_{b}^{0} / m_{r}\right)\left(1-r^{2} / R_{C}^{2}\right)$, with $n_{b}^{0}$ the condensate density in the origin and $R_{C}$ the radius of the condensate. This results in an additional confinement potential (inverted for $a_{\text {scatt }}>0$ ) acting on the fermions. For the resonance used above, for $n_{b}^{0}=10^{14} \mathrm{~cm}^{-3}$ and $R_{C}=10 \mu \mathrm{m}$ and making use of Eq. (1), its frequency equals $\omega_{b f}=4.95 \times 10^{3} \mathrm{~s}^{-1} / \sqrt{\tilde{\nu}}$. For $\tilde{\nu}$ not too small, this is much lower than the frequency scale of the molecule formation $g_{m} \sqrt{n_{b}^{0}} / \hbar=7.72 \times 10^{5} \mathrm{~Hz}$, which means that the dynamics due to molecule formation is fast compared to the mean-field collapse, and the local-density approximation can be used. For $\tilde{\nu}=1$, an atom migrates $10 \%$ of the trap length in $0.75 \mathrm{~ms}$, whereas the period of a Rabi oscillation is around $1.3 \mu \mathrm{s}$.

Within the approximations described above, we plot in Fig. 4 the density of slow fermions as a function of time and distance from the center of the trapping potential in the case that the fermion cloud has a radius 1.5 times larger than the condensate. To use the formalism presented above, space
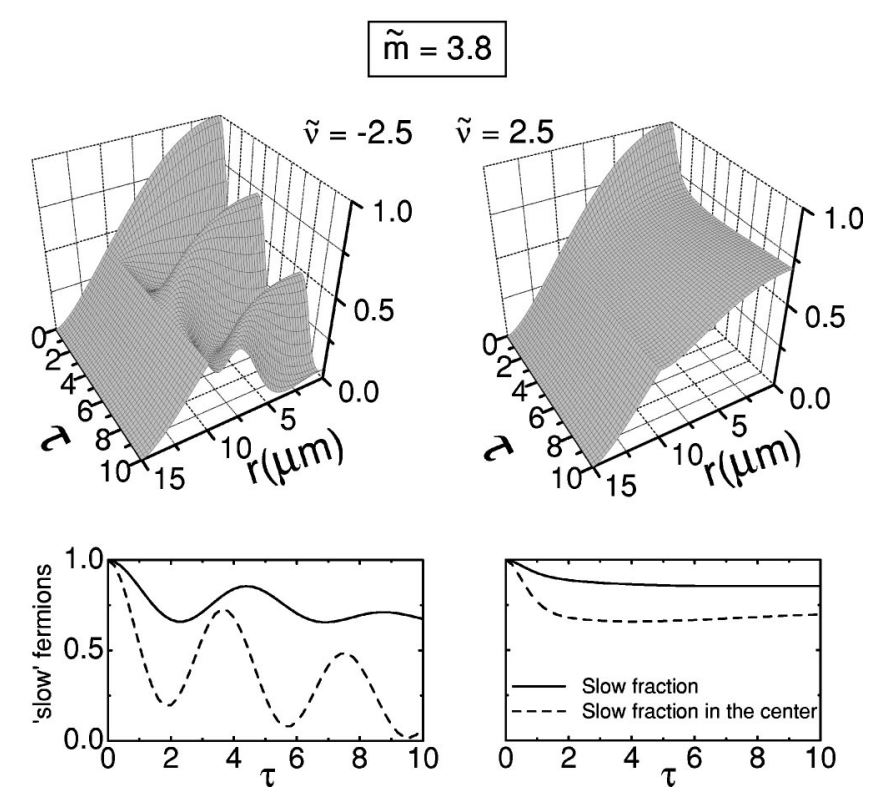

FIG. 4. The time evolution of the density of slow fermionic atoms, normalized such that the density at $t=0$ equals 1 in the center of the trap, is shown in the upper panels. At time $t=0$, the noninteracting boson-fermion mixture in a harmonic trap is suddenly brought close to a Feshbach resonance. In the lower panels, the spatially averaged fraction of slow fermionic atoms is compared with this fraction in the center of the trap. The inhomogeneity decreases the spatially averaged oscillations because the amplitude and frequency depend on the condensate density and consequently vary in space. The parameters used are the same as for the right panels in Fig. 3, $\tilde{m}=3.8$ and $\tilde{\nu}= \pm 2.5$, and correspond to a realistic situation in ${ }^{40} \mathrm{~K}-{ }^{87} \mathrm{Rb}$ mixtures.

was subdivided in to discrete boxes in which the gases were presumed homogeneous, as in the local density approximation. As discussed above, no redistribution of fermions across volume elements is considered so that each volume element can be treated independent of the others. In the lower panels, the total fraction, averaged over space, of slow fermionic atoms is compared to the local fraction of slow fermionic atoms in the center of the trap. One can see that the inhomogeneity decreases the amplitude of the oscillations in the total fraction of slow fermionic atoms. The reason is that the condensate is the most dense in the middle, which increases the efficiency of the atom-molecule conversion, and also because $\tau, \tilde{m}$, and $\tilde{\nu}$ depend on $n_{b}(r)$, so that on different places in the condensate oscillations with different frequencies occur.

\section{CONCLUSIONS}

We have made a study of the dynamics of a fermionboson mixture resulting from a fast switch in the magnetic field, bringing it close to an interspecies Feshbach resonance. Our analysis shows that damped oscillations between fermionic atoms and molecules will occur on the typical time 
scale of $\hbar /\left(g_{m} \sqrt{n_{b}}\right)\left(\approx 1 \mu\right.$ s for a ${ }^{40} \mathrm{~K}-{ }^{87} \mathrm{Rb}$ mixture $)$. Such types of oscillations have recently been observed in singlecomponent BEC's near a Feshbach resonance [4]. The decay of the molecules in pairs of fast fermionic atoms and bosons leads to heating of the system, but a core of slow fermions will remain due to the existence of a bound state of the fermions coupled to the condensate and the continuum of thermal bosons. Using the latest data on resonances in ${ }^{40} \mathrm{~K}-{ }^{87} \mathrm{Rb}$ scattering $[12,13]$, we find that the predicted oscillations between slow fermions and slow molecules should be observable both in a homogeneous and an inhomogeneous system. The redistribution of the densities are expected to occur much slower than these oscillations.

\section{ACKNOWLEDGMENTS}

The authors would like to thank G. Modugno for useful and stimulating discussions and A. Simoni for providing us with the latest theoretical predictions on Feshbach resonances in ${ }^{40} \mathrm{~K}-{ }^{87} \mathrm{Rb}$. Discussions with $\mathrm{M}$. Inguscio are also gratefully acknowledged. Two of the authors (M.W. and J.T.) were supported financially by the Fund for Scientific Research-Flanders (Fonds voor Wetenschappelijk Onderzoek-Vlaanderen). This research was supported financially by the FWO-V through Project Nos. G.0435.03 and G.0306.00, the WOG through Project No. WO.025.99N, and the GOA BOF UA 2000, IUAP.
[1] A.G. Truscott, K.E. Strecker, W.I. McAlexander, G.B. Partridge, and R.G. Hulet, Science 291, 2570 (2001).

[2] F. Schreck, L. Khaykovich, K.L. Corwin, G. Ferrari, T. Bourdel, J. Cubizolles, and C. Salomon, Phys. Rev. Lett. 87, 080403 (2001).

[3] G. Modugno, G. Roati, F. Riboli, F. Ferlaino, R.J. Brecha, and M. Inguscio, Science 297, 2240 (2002).

[4] E.A. Donley, N.R. Clausen, S.T. Thompson and C.E. Wieman, Nature (London) 417, 529 (2002).

[5] S.J.J.M.F. Kokkelmans and M.J. Holland, Phys. Rev. Lett. 89, 180401 (2002).

[6] T. Koehler, T. Gasenzer, and K. Burnett, Phys. Rev. A 67, 013601 (2003).

[7] R.A. Duine and H.T.C. Stoof, e-print cond-mat/0210544.
[8] M. Mackie, K.A. Suominen, and J. Javanainen, Phys. Rev. Lett. 89, 180403 (2002).

[9] E. Timmermans, P. Tommasini, R. Côté, M. Hussein, and A. Kerman, Phys. Rev. Lett. 83, 2691 (1999).

[10] S.J.J.M.F. Kokkelmans, J.N. Milstein, M.L. Chiofalo, R. Walser, and M.J. Holland, Phys. Rev. A 65, 053617 (2002).

[11] K. Rzazewski, M. Lewenstein, and J.H. Eberly, J. Phys. B 15, L661 (1982).

[12] A. Simoni, G. Modugno, G. Roati, and M. Inguscio, e-print cond-mat/0301159.

[13] A. Simoni (private communication).

[14] C. J. Pethick and H. Smith, Bose-Einstein Condensation in Dilute Gases (Cambridge University Press, Cambridge, 2002), p. 138. 